\title{
Transposição de grandes vasos: uma comparação entre as técnicas operatórias
}

\author{
Great vessels transposition: a comparison among the surgical techniques
}

Transposición de grandes vasos: una comparación entre las técnicas cirúrgicas

Laura Cattony Pimenta ${ }^{1 *}$, Victor Hugo Duarte Pimenta1, João Luiz de Pinho Tavares Tristão', Sofia Helena Rugeri Pimenta ${ }^{1}$, Alice Cesário da Silva ${ }^{1}$, Luan Cabral Santana ${ }^{1}$, Pedro Henrique Lopes Macedo ${ }^{1}$, Ewerton Ribeiro Maciel ${ }^{1}$, Fernando Augusto de Vasconcellos Santos ${ }^{1}$.

\section{RESUMO}

Objetivo: Discutir e sintetizar os aspectos da transposição, enumerando os sinais e sintomas, métodos diagnósticos e tratamentos, além de descrever a aplicabilidade, a técnica utilizada e possíveis evoluções da correção cirúrgica. Revisão Bibliográfica: Foi realizada uma revisão literária narrativa com busca nas bases de dados Scielo, Public/Publisher MEDLINE (PubMed), National Center for Biotechnology Information (NCBI) e Springer, entre os anos 1981 e 2020, com os descritores 'Transposição de Grandes Vasos", "Transposição de Grandes Artérias", "Técnicas operatórias TGV", "Arterial Switch Operation" e "Cirurgia de Lecompte". Esta entidade patológica engloba múltiplas alterações anatômicas que podem acompanhar a inversão na posição das grandes artérias, influenciando a apresentação clínica. É a principal causa de procedimentos cirúrgicos neonatais, com diferentes técnicas que se adequam de acordo com o estado do paciente. O diagnóstico só é confirmado por exames de imagem. Considerações finais: A TGV, se não acompanhada de shunt sanguíneo, é incompatível com a vida. Portanto, requer estabilização hemodinâmica e monitorização cuidadosa, até que seja eleito o método cirúrgico mais adequado para restaurar a comunicação entre fluxos sistêmico e pulmonar.

Palavras-chave: Transposição de grandes vasos, Transposição de grandes artérias, Comunicação interventricular, Obstrução da via de saída do ventrículo esquerdo.

\begin{abstract}
Objective: Discuss and synthesize the aspects of this heart disease, listing the signs and symptoms, diagnostic methods and treatments, in addition to describing the applicability, the technique used and possible evolutions of the surgical correction. Bibliographic review: A narrative literary review was carried out using the Scielo, Public/Publisher MEDLINE (PubMed), National Center for Biotechnology Information (NCBI) and Springer databases, between the years 1981 and 2020, using as keywords 'Transposition of Great Vessels", "Transposition of Great Arteries", "TGV Surgical Techniques", "Arterial Switch Operation" and "Lecompte Procedure". This pathological entity includes multiple anatomical changes that can accompany the inversion in the position of the great arteries, influencing clinical presentation. It is the main cause of neonatal surgical procedures, with different techniques that are adapted according to the patient's condition. The diagnosis is only confirmed by image exams. Final considerations: TGV, if not accompanied by blood shunt, is incompatible with life. Therefore, it requires hemodynamic stabilization and careful monitoring, until the most appropriate surgical method is chosen to restore communication between systemic and pulmonary flows. The diagnosis is only confirmed by image exams.
\end{abstract}

Keywords: Transposition of great vessels, Transposition of great arteries, Interventricular communication, Left ventricular outflow tract obstruction.

\footnotetext{
${ }^{1}$ Faculdade Ciências Médicas de Minas Gerais, Belo Horizonte - MG. *E- mail: lauracattonyy@gmail.com
} 


\section{RESUMEN}

Objetivo: Discutir y sintetizar los aspectos de esta cardiopatía, enumerando los signos y síntomas, métodos diagnósticos y tratamientos, además de describir la aplicabilidad, la técnica empleada y las posibles evoluciones de la corrección quirúrgica. Revisión bibliográfica: Se realizó una revisión literaria narrativa utilizando las bases de datos Scielo, Public/Publisher MEDLINE (PubMed), National Center for Biotechnology Information (NCBI) y Springer, entre los años 1981 y 2020, con las palavras-clave "Transposición de vasos grandes", "Transposición de grandes arterias", "Técnicas operativas de TGV", "Operación de interruptor arterial" y "Cirugía de Lecompte". Esta entidad patológica incluye múltiples cambios anatómicos que pueden acompañar a la inversión en la posición de las grandes arterias, influyendo en la presentación clínica. Es la principal causa de procedimientos quirúrgicos neonatales, con diferentes técnicas que se adaptan según el estado del paciente. El diagnóstico solo se confirma con pruebas de imagen. Consideraciones finales: Si el TGV no se acompaña de derivación sanguínea, es incompatible con la vida. Por tanto, requiere una estabilización hemodinámica y una monitorización cuidadosa, hasta que se elija el método quirúrgico más adecuado para restablecer la comunicación entre los flujos sistémico y pulmonar.

Palabras-clave: Transposición de vasos grandes, Transposición de grandes arterias, Comunicación interventricular, Obstrucción del tracto de salida del ventrículo izquierdo.

\section{INTRODUÇÃO}

Segundo Marathe SP (2015), transposição de grandes vasos (TGV), ou transposição de grandes artérias, é uma importante cardiopatia cianótica entre recém-nascidos, representando cerca de $5 \%$ do total de anomalias cardíacas congênitas. Sem um mecanismo completamente descrito, a anomalia foi relatada morfologicamente pela primeira vez pelo médico inglês Matthew Baillie (1797) e caracteriza-se pela inversão de posições das grandes artérias do coração: aorta e pulmonar. Nestes casos, a primeira encontra-se no ventrículo direito e a segunda, no esquerdo, um espelho patológico da anatomia normal. Com isso, formamse duas circulações distintas, com o sangue sistêmico chegando ao ventrículo direito e retornando para a aorta em direção à circulação sistêmica, e o sangue oxigenado da circulação pulmonar chegando ao ventrículo esquerdo apenas para ser bombeado novamente aos pulmões.

Como encontrado no artigo de Binotto CN (2018) múltiplas apresentações morfológicas dessa cardiopatia, podendo ou não envolver outras alterações estruturais, como comunicação interventricular (CIV) e obstrução da via de saída do ventrículo esquerdo (OVSVE). Além disso, raramente a aorta apresenta-se posteriormente à artéria pulmonar. Dessa maneira, há que se diferenciar a TGV clássica, quando não ocorrem estas outras anomalias, da complexa, que pode ser clinicamente mais branda e, por isso, mais difícil de se identificar. Embora a etiologia seja ainda desconhecida, acredita-se que seja multifatorial e envolva fatores de risco que aumentem a incidência, como a mãe ser portadora de diabetes mellitus (DM).

A mudança na posição entre a artéria aorta e o tronco pulmonar ocasiona desorganização no sistema circulatório corporal. Como a aorta sai do ventrículo direito, o sangue direcionado à grande circulação é venoso, com baixo teor de oxigênio. Por isso, não há perfusão adequada dos tecidos corporais, com destaque para as vísceras nobres, como cérebro, pulmão, rins, pâncreas e o próprio coração. Assim, explica-se a apresentação clínica de cianose e a gravidade do quadro (WRENC C, et al., 2003).

Como encontrado, até o momento, o diagnóstico dessa anomalia congênita é comumente realizado em período pós-natal. Não existe consenso no meio científico acerca da viabilidade prática de diagnóstico prénatal. É desafiador, devido às restrições no estudo hemodinâmico da circulação cardiopulmonar em fetos, não só pelas pequenas dimensões das câmaras cardíacas, mas também pelas limitações técnicas observadas na ultrassonografia percutânea. Então, embora seja possível identificar com acurácia a alteração na posição arterial, rotineiramente não é comum, e, então, o diagnóstico pós-natal é muito mais frequente (SZYMANSKI MW, et al., 2020).

Segundo Zielinsky P (1997), o recém-nascido classicamente apresenta cianose extrema, com baixa saturação de oxigênio tanto proximal quanto distal ao ducto arterioso. Nesses casos, está indicado o prosseguimento da propedêutica com ecocardiograma e radiografia de tórax. 
Como encontrado no artigo de Pedra SRFF (2019) logo após o nascimento, a estabilização hemodinâmica imediata é feita com a infusão contínua, por cateter umbilical venoso, de prostaglandina E1. Este procedimento visa à manutenção da perviedade do ducto arterioso, uma comunicação interventricular própria da circulação fetal que, sem o uso destes potentes vasodilatadores, fecharia pouco tempo após o nascimento. Aberta, esta estrutura permite que parte do sangue oxigenado presente no ventrículo esquerdo passe para 0 ventrículo direito, de onde será bombeado através da aorta para atingir a circulação sistêmica. Assim, procurase garantir a vida, embora o tratamento definitivo seja obrigatoriamente cirúrgico. A técnica operatória utilizada é escolhida individualmente, de acordo com o número de alterações existentes e as condições do coração.

Por fim, o objetivo deste trabalho é discutir e sintetizar os aspectos da transposição, enumerando os sinais e sintomas, métodos diagnósticos e tratamentos, além de descrever a aplicabilidade, a técnica utilizada e possíveis evoluções da correção cirúrgica.

\section{REVISÃO BIBLIOGRÁFICA}

Segundo Shima Y, et al. (2011), a prevalência da TGV é de 4.7 a cada 10.000 nascidos vivos, correspondendo de 3 a $5 \%$ das cardiopatias congênitas e até $20 \%$ das cardiopatias cianóticas. Além disso, é duas vezes mais comum no sexo masculino em relação ao feminino. As crianças acometidas nascem com tamanho dentro dos limites de normalidade.

Como encontrado no artigo de Binotto CN, et al. (2018), o mecanismo de desenvolvimento da TGV ainda não é totalmente conhecido, embora seja discutida a absorção dos cones sub-aórtico e sub-pulmonar como causa da anatomia invertida. No desenvolvimento normal, entre 30 e 34 dias de gestação, há a absorção do cone sub-aórtico, permitindo a rotação da aorta posteriormente ao tronco pulmonar e sobre o átrio esquerdo, sua posição anatômica, e levando à continuidade das valvas mitral e aórtica, enquanto o cone sub-pulmonar não é absorvido, o que mantém o tronco pulmonar no ventrículo direito. Na TGV, porém, há uma inversão desses fatores, com o cone sub-pulmonar sendo absorvido, enquanto não há absorção do cone sub-aórtico. Isso leva a uma movimentação posterior do tronco arterial e a criação de uma continuidade entre valva pulmonar e mitral. Já o cone sub-aórtico persistente causa a migração anterior da aorta, para dentro do átrio direito.

Segundo Zielinsky P (1997) a resultante dessa anatomia é a criação de duas circulações independentes, a sistêmica e a pulmonar. Nela, o sangue desoxigenado da circulação sistêmica retorna ao ventrículo direito e é direcionada novamente para ela, enquanto o sangue oxigenado da circulação pulmonar chega ao ventrículo esquerdo apenas para ser bombeado novamente para os pulmões.

Segundo Shima Y, et al. (2011), durante a vida intrauterina, não há obstáculos ao desenvolvimento do concepto, já que, na circulação fetal, o sangue é oxigenado pelas vilosidades coriônicas da placenta, e se desvia dos pulmões, que possuem alta resistência, através do forame oval e do ducto arterioso. Dessa forma, chega ao ventrículo direito e é diretamente bombeado para a aorta em direção aos tecidos periféricos.

No entanto, ao nascimento, a primeira inspiração causa vasodilatação das artérias e queda da resistência pulmonar ao fluxo sanguíneo, e a perda do cordão umbilical acaba com a chegada de sangue oxigenado proveniente da mãe. A partir desse momento, o sangue dos pulmões, que passa a ser responsável pela oxigenação do organismo, é impedido de atingi-lo. Por isso, inicia-se um quadro de cianose progressiva: a cada batimento cardíaco, o sangue tem a sua oxigenação reduzida pelo metabolismo aeróbio tecidual até que se tornaria incompatível com a vida, a não ser pela presença de um shunt, que pode ser atingido pela manutenção do ducto arterioso (SHIMA Y, et al., 2011).

Como encontrado no artigo de Zielinsky P (1996), caracterizada apenas pela inversão de posição entre as grandes artérias cardíacas, a TGV é chamada clássica ou simples. Entretanto, pode haver outras anomalias morfológicas, como comunicação interventricular (CIV), comunicação interatrial (CIA), estenose pulmonar e obstrução da via de saída do ventrículo esquerdo (OVSVE), que levam à TGV complexa. O shunt da esquerda para a direita promovido pela CIV, sendo o VE hipertrófico em comparação com o VD, exerce papel no alívio da cianose, assim como CIA. Por outro lado, a OVSVE e a estenose pulmonar promovem hipertrofia patológica do VE. 
Segundo Zielinsky P (1997), a forma clássica, o paciente apresentará cianose progressiva nas primeiras horas de vida e hiperfonese de B2 única em foco pulmonar. É variável a presença de sopro cardíaco, que, quando existente, será discreto e mais bem identificado em foco tricúspide ou pulmonar. A forma complexa, por sua vez, apresenta-se com cianose discreta, em função do shunt proporcionado pelas anomalias adjuntas. Neste caso, o paciente poderá apresentar sinais de insuficiência cardíaca cerca de um mês após o nascimento, devido ao aumento do fluxo pulmonar.

Como encontrado no artigo de Binotto $\mathrm{CN}$, et al. (2018), a apresentação da radiografia pode estar normal ou conter alterações sugestivas, tais como: formato cardíaco oval, cardiomegalia mediana ou aumento das marcações vasculares pulmonares. Entretanto, o ecocardiograma é o padrão-ouro para que o diagnóstico seja fechado. Além disso, ressalta-se a importância de se utilizarem as informações obtidas nesse exame durante a cirurgia, sendo as principais: diâmetros da aorta e da artéria pulmonar, o local da comissura das válvulas e a origem e trajeto das coronárias.

Segundo Sarris G, et al. (2017), principal impacto causado no paciente pela TGV é oriundo do shunt, resultado da alteração da anatomia normal dos vasos do coração ou da comunicação interatrial, cuja presença determina controle mais hemodinâmico mais complexo. Nesse contexto pré-operatório, é de vital importância a estabilização do paciente visando o controle hemodinâmico e respiratório. Destaca-se que a não responsividade ao oxigênio ofertado por meio de catéteres e máscaras faciais é uma característica desta patologia.

Como encontrado no artigo de Bubols GB, et al. (2014), para que as manifestações clínicas nas primeiras horas de vida sejam minimizadas, é imperativa a administração de prostaglandinas E1 (PGE1) por via intravenosa para que o ducto arterioso se mantenha pérvio. Concomitantemente, oxigênio suplementar é administrado, a fim de que a perfusão capilar e a saturação se aproximem dos valores adequados, corrigindo, assim, a acidose metabólica gerada pelo processo patológico.

Segundo Sarris G, et al. (2017) pode-se destacar a dificuldade do manejo hemodinâmico nos pacientes que apresentam a comunicação interatrial restritiva, sendo que a administração de PGE1 pode não ser suficiente para corrigir as alterações provocadas pelo shunt patológico. Dessa forma, esses recém-nascidos são encaminhados para uma septostomia atrial de emergência com continuidade da PGE1, procedimento que requer o uso de paracetamol, fentanil e benzodiazepínicos. Além disso, a dexmedetomidina tem ganhado espaço nesses casos, por não apresentar efeitos depressivos no sistema respiratório.

Como encontrado no artigo de Bubols GB, et al. (2014) o tratamento com PGE1 ou a septostomia atrial, espera-se que o shunt seja controlado em nível atrial, o que justifica o fato de a PGE1 ser comumente descontinuada, o que muitas vezes leva a efeito rebote de hipoxemia. Entretanto, a existência de coarctação de aorta, ou qualquer outra obstrução atrial, interfere na resposta terapêutica esperada com a infusão de PGE1, podendo impedir sua interrupção. Dessa maneira, alguns médicos optam pelo desmame gradual, recomendado para pacientes que passaram pela septostomia atrial. Devido aos contratempos que pode engatilhar, a retirada deve ser feita de forma planejada, mas precisa ser executada, uma vez que a continuidade do medicamento pode gerar efeitos adversos, e a manutenção da patência do ducto arterioso pode levar a um quadro de edema pulmonar.

Alguns pacientes podem não estabilizar mesmo após a septostomia atrial, mantendo a acidemia e a cianose. Para esses casos, é recomendada a realização de ecocardiograma para definir, principalmente, se há presença de hipertensão pulmonar, cujo quadro persistente é mais comum em pacientes com a comunicação interatrial (SARRIS G, et al., 2017).

Segundo Bubols GB, et al. (2014), além dos tratamentos convencionais, alguns outros estão descritos na literatura, majoritariamente na forma de relato de casos, como o uso de hiperventilação para a estabilização da saturação do paciente e correção da acidose, de óxido nítrico inalatório, que leva à dilatação da circulação pulmonar e combate a hipertensão persistente, de sildenafil, o qual atua aumentando a liberação de óxido nítrico para reduzir os níveis pressóricos pulmonares, e de bosentan, medicamento antagonista duplo do receptor de endotelina indicado para o tratamento de hipertensão pulmonar e suporte de vida extracorpóreo. 
Primeira operação realizada, com bons resultados, para a correção da TGA foi implementada pelo Doutor Rastelli, nome pelo qual ficou conhecida. Apesar de bons resultados imediatos e tardios e de ser utilizada até os dias atuais, se desenvolveram técnica operatória de maior reprodutibilidade e melhores resultados clínicos, sendo a técnica de escolha na maioria dos centros mundiais (SARRIS G, et al., 2017).

Cirurgia de Rastelli é um procedimento cirúrgico que visa corrigir TGA, CIV e obstrução da via de saída do ventrículo esquerdo (OVSVE) por meio do uso de próteses extracardíacas que formam túneis de comunicação entre o ventrículo esquerdo e a aorta e entre o ventrículo direito e a artéria pulmonar. Apesar dos bons resultados imediatos alcançados com essa abordagem cirúrgica, questiona-se muito seus resultados a longo prazo e critica-se a sua aplicabilidade, levando-se em consideração sua complexidade técnica (SARRIS G, et al., 2017).

Pode-se destacar que não está indicada na maioria dos casos devido ao padrão de variação anatômica do sistema cardiovascular, que está contraindicada para pacientes neonatos e lactentes devido à alta prevalência de obstrução da prótese extracardíaca nessa faixa etária, que necessita de troca da prótese em médio prazo devido ao processo de degeneração da mesma, que nem sempre o mediastino é capaz de comportar a prótese e essa pode ser obstruída por compressão do esterno, que há casos de isquemia miocárdica devido à compressão das artérias coronárias pelo anel da prótese valvar (SARRIS G, et al., 2017) .

Por outro lado, a operação à Lecompte apresenta maior aplicabilidade tanto para variações anatômicas intracardíacas quanto para as valvares, além disso não faz uso de próteses evitando, assim, os problemas tardios citados anteriormente. Entretanto, mesmo sendo aplicada para número maior gama de malformações, seu uso é restrito quando se trata de CIV múltipla ou distante da valva aórtica e hipoplasia ventricular ou arterial. Nesse contexto, pode-se complementar os benefícios evidenciados no pós-operatório, tais como: menor gradiente intratúnel que liga ventrículo esquerdo e aorta e menor incidência de reoperações. Por fim, vale ressaltar que a técnica introduzida por Lecompte é indicada para neonatos e lactantes, além de ser indicada para pacientes que não tiveram boa evolução após realizada a cirurgia de Rastelli (SARRIS G, et al., 2017).

Válido citar que existem outras técnicas operatórias com o objetivo de contornar os efeitos deletérios da TGV, porém essas são de aplicação reduzida e possuem valor histórico, tais com as técnicas segundo Mustard e Senning, cirurgia de Nakaidoh, cirurgia de reparação ventricular à l'Etage e cirurgia de Yasui. Não obstante, a técnica operatória proposta por Jatene et al., cuja grande complexidade determina a dependência da habilidade do cirurgião, é atualmente considerada como tratamento definitivo de escolha para a TGV, já que apresenta boa evolução clínica e sobrevida de até 96\% (MALUF MA, et al., 2006).

Embora o tratamento cirúrgico proporcione correção completa, ocasionais lesões residuais podem permanecer. Isso se deve à escolha inadequada da técnica ou, mais comumente, à própria condição da anatomia cardíaca em questão. Assim, o paciente pode apresentar arritmias supraventriculares, insuficiência tricúspide, disfunção de VD ou estenose pulmonar, o que pode significar necessidade de reintervenção cirúrgica (SARRIS G, et al., 2017).

Apesar da elevada quantidade de paciente abordados pela técnica de Lecompte, além da menor de taxa de morbidade e mortalidade desta, deve-se levar em consideração que é uma técnica desenvolvida em 1981 e necessita de maiores aprimoramentos. Sendo assim, nos dias atuais novas drogas e mudanças de etapas da técnica tem sido abordada afim de descobrir uma maneira mais eficiente e segura da aplicação da cirurgia de Lecompte. Dentre os piores dados gerados pela operação podemos destacar a necessidade de reoperação em pacientes que passaram pelo procedimento (SARRIS G, et al., 2017).

Uma publicação recente da Universidade de Cambrigde mostrou que a maior incidência de reoperação acontece no primeiro ano após o tratamento cirúrgico, e que essa taxa reduz progressivamente com o passar dos anos entre as reoperações tardias são infrequentes e, quando são necessárias, mais que $80 \%$ delas acontecem antes dos 10 anos após a primeira intervenção. Por fim, podemos destacar que uma reintervenção na vida adulta de um paciente que já foi submetido a uma durante a infância tende a ter altos riscos de morbimortalidade (SARRIS G, et al., 2017). 
Dentre as principais causas de reoperação em pacientes submetidos à operação de Lecompte para tratamento de TGA temos: obstrução do fluxo da artéria pulmonar, regurgitação da valva pulmonar, regurgitação de valva aortica, obstrução do fluxo da aorta, lesão de artéria coronária (a esquerda é mais afetada), obstrução traqueobronquial e esofágica por estruturas vasculares e regurgitação residual da valva mitral (PEDRA SRFF, et al., 2019).

Em contrapartida pode-se destacar que $80 \%$ dos pacientes submetidos ao tratamento cirúrgico não terão que passar por uma outra intervenção em até 20 anos. Além disso, a necessidade de uma reoperação não interfere expressivamente nos dados de morbimortalidade dos pacientes. Sendo assim, podemos destacar que as inovações almejadas pelos cirurgiões tendem a impedir que um quinto dos pacientes sejam submetidos a novos estresses e a diminuir as taxas de morbimortalidade da técnica ainda mais (PEDRA SRFF, et al., 2019).

Ao revermos a literatura sobre a TGA, pode-se concluir que é uma doença que carrega em si diversas alterações morfológicas em sítio cardíaco para o neonato e lactante. Dessa forma, é possível associar as existentes taxas de morbimortalidade com as imprevisíveis malformações do paciente que, às vezes, só são reconhecidas durante a operação ou no post mortem em exame de necrópsia. O tratamento cirúrgico faz-se necessário nesses casos e, usualmente, é precedido pela infusão de PGE1 para a estabilização hemodinâmica e correção dos distúrbios ácidos-básicos sanguíneos. A intervenção cirúrgica proposta por Lecompte é técnica reprodutível pela maioria dos cirurgiões e a mais aplicada mundialmente. Jatene et al. propuseram técnica operatória com excelentes resultados a despeito de ser complexa e demandar grande expertise por parte dos cirurgiões (SARRIS G, et al., 2017)..

Como encontrado no artigo de Jatene MB, et al. (2005), nesse contexto, pode-se citar o artigo publicado por Lecompte J, et al. (1991), no qual o grupo descreve uma nova técnica operatória, porém, relata que três pacientes evoluíram para óbito dentre os nove abordados, sendo que todos os pacientes tinham TGA e defeito no septo ventricular. É descrito que um paciente morreu por uma grave estenose da artéria pulmonar resultante de uma hipoplasia da valva aórtica, um paciente morreu por iatrogenia durante o pós-operatório e outro paciente morreu devido a uma coarctação de aorta "irreconhecível" e uma anormalidade da valva tricúspide. Portanto, conclui-se que a falta de êxito cirúrgico normalmente é associado a dificuldades de abordagem reconstrutiva e não ao uso da técnica descrita por Lecompte J, et al. (1991).

\section{CONSIDERAÇÕES FINAIS}

É notória a relevância desta cardiopatia no âmbito da clínica, da cirurgia pediátrica e da cirurgia cardiovascular. Faz-se essencial, portanto, que o treinamento médico capacite os profissionais para realização do diagnóstico precoce e escolha da conduta mais apropriada, de forma individualizada para cada paciente. Nesse sentido, o conhecimento acerca da estabilização hemodinâmica imediata e do procedimento cirúrgico adequado pode influenciar nas taxas de morbimortalidade da doença. Após consultar a literatura que descreve as técnicas para a abordagem da TGA, fica evidente que a operação à Lecompte apresenta menores taxas de morbimortalidade.

\section{REFERÊNCIAS}

1. ATIK E, et al. Lactente de 7 meses, do sexo feminino, com transposição das grandes artérias e comunicação interventricular subpulmonar (anomalia de Taussig-Bing). Arquivos Brasileiros de Cardiologia, 2010; 95(1):1-3.

2. BINOTTO CN, et al. Transposição de grandes vasos em criança de 1 ano de idade. Resid Pediatr, 2018; 8(1): 41-44.

3. BUBOLS GB, et al. Nitric oxide and reactive species are modulated in the polyphenol-induced ductus arteriosus constriction in pregnant sheep. Prenat Diagn, 2014; 34(13) :1268-76.

4. CUNEO BF, et al. Prenatal diagnosis and in utero treatment of torsades de pointes associated with congenital long QT syndrome. Am J Cardiol, 2003; 91(11): 1395-8.

5. DEDEMOGLU M, et al. Modified Closed Coronary Transfer is a Good Alternative to the Trap-Door Method During Arterial Switch Operation: A Retrospective Propensity-Matched Comparison. Braz J Cardiovasc Surg, 2020; 35(3): 329-38.

6. GARDINER HM. Progression of fetal heart disease and rationale of fetal intracardiac interventions. Semin Fetal Neonatal Med, 2005; 10(6): 578-85. 
7. HOFFMAN JI, CHRISTIANSON R. Congenital heart disease in a cohort of 19,502 births with long-term follow-up. Am J Cardiol., 1978; 42(4): 641-7.

8. JATENE MB, et al. Correção cirúrgica da transposição das grandes artérias: 30 anos de operação de Jatene. Revista Médica de São Paulo, 2005; 84(3-4): 113-7.

9. JARVIS K, et al. Hemodynamic evaluation in patients with transposition of the great arteries after the arterial switch operation: 4D flow and 2D phase contrast cardiovascular magnetic resonance compared with Doppler echocardiography. Journal of Cardiovascular Magnetic Resonance, 2016; 18(59): 1-9.

10. KOMAROV R, et al. State-of-the-Art Pediatric Coronary Artery Bypass Surgery: a Literature Review. Braz J Cardiovasc Surg, 2020; 35(4): 539-48.

11. LECOMPTE, Y, et al. Réparation á l'etage ventriculaire - the REV procedure: technique and clinical results. Cardiol Young, 1991; 1(1): 63-70.

12. MALUF MA, et al. Procedimento de Lecompte para a correção de transposição das grandes artérias, associada à comunicação interventricular e obstrução de via de saída do ventrículo esquerdo. Braz J Cardiovasc Surg, 2006; 21(4): 433-43.

13. MARATHE SP, TALWAR S. Surgery for transposition of great arteries: A historical perspective. Ann Pediatr Cardiol, 2015; 8(2): 122-128.

14. MOISE KJ, et al. Indomethacin in the treatment of premature labor. Effects on the fetal ductus arteriosus. $\mathrm{N}$ Engl $\mathrm{J}$ Med, 1988; 319(6): 327-31

15. PEDRA SRFF, et al. Diretriz Brasileira de Cardiologia Fetal - 2019. Arquivos Brasileiros de Cardiologia, 2019; 112(5): 600-48.

16. SARRIS G, et al. Clinical guidelines for the management of patients with transposition of the great arteries with intact ventricular septum: The Task Force on Transposition of the Great Arteries of the European Association for CardioThoracic Surgery (EACTS) and the Association for European Pediatric and Congenital Cardiology (AEPC). Cardiology in the Young, 2017; 27(3), 530-69.

17. SHIMA Y, et al. Idiopathic severe constriction of the fetal ductus arteriosus: a possible underestimated pathophysiology. Euro J Pediatr, 2011; 170(2): 237-40.

18. SIMPSON LL, et al. Nuchal translucency and the risk of congenital heart disease. Obstet Gynecol, $2007 ; 109(2 \mathrm{Pt} 1)$ : 376-83.

19. SZYMANSKI MW, et al. Transposition of The Great Arteries. [Updated 2021 Jan 21]. In: StatPearls [Internet]. Treasure Island (FL): StatPearls Publishing; 2020.

20. WREN C, et al. Cardiovascular malformations in infants of diabetic mothers. Heart, 2003; 89(10): 1217-20.

21. ZIELINSKY P. Fetal heart rhythm disorders: Detection and prenatal management. Arquivos Brasileiros de Cardiologia, 1996; 66(2): 83-6. 